\title{
Effective Incorporation of Spatial Information in a Mutual Information Based 3D-2D Registration of a CT Volume to X-Ray Images
}

\author{
Guoyan Zheng \\ MEM Research Center, University of Bern, Stauffacherstr. 78, CH-3014, Switzerland \\ guoyan.zheng@ieee.org
}

\begin{abstract}
This paper addresses the problem of estimating the 3D rigid pose of a $\mathrm{CT}$ volume of an object from its $2 \mathrm{D}$ X-ray projections. We use maximization of mutual information, an accurate similarity measure for multi-modal and mono-modal image registration tasks. However, it is known that the standard mutual information measure only takes intensity values into account without considering spatial information and its robustness is questionable. In this paper, instead of directly maximizing mutual information, we propose to use a variational approximation derived from the Kullback-Leibler bound. Spatial information is then incorporated into this variational approximation using a Markov random field model. The newly derived similarity measure has a least-squares form and can be effectively minimized by a multi-resolution LevenbergMarquardt optimizer. Experimental results are presented on X-ray and CT datasets of a plastic phantom and a cadaveric spine segment.
\end{abstract}

\section{Introduction}

3D-2D registration of a three-dimensional (3D) CT volume with two-dimensional (2D) X-ray images has shown great potential in various applications including intra-operative patient pose estimation and post-operative prosthesis alignment evaluation. The reported techniques to achieve this registration can be split into two main categories: feature-based methods and intensity-based methods. Feature-based methods require a prerequisite segmentation stage which is errorprone and hard to achieve automatically. The errors in segmentation can lead to errors in the final registration. In contrast, intensity-based methods directly compare the X-ray image with the associated digitally reconstructed radiograph (DRR), which is obtained by simulating X-ray projection of the CT volume. No segmentation is required.

In this work, we use maximization of mutual information (MI), an accurate similarity measure for multi-modal and mono-modal image registration tasks [1] 2] 3. However, it is known that the standard mutual information measure only takes intensity values into account without considering spatial information and its robustness is questionable [4]. 
Several attempts have been made to adapt the MI-based registration framework to incorporate spatial information of individual images [4 [5] 6] 7] 8]. However, the resultant similarity measure either requires computing the entropy of higher dimensional probability distributions, which is not advisable because of the increase of statistical uncertainties with higher dimensions due to the scarcity of data, or is not robust to outliers.

In this paper, instead of directly maximizing mutual information, we propose to use a variational approximation derived from the Kullback-Leibler bound 9]. Spatial information is then incorporated into this variational approximation using a Markov random field (MRF) model [10. The newly derived similarity measure has a least-squares form and can be effectively minimized by a multiresolution Levenberg-Marquardt non-linear optimizer.

\section{The Proposed Approach}

\subsection{Derivation of a Variational Approximation to the MI}

In this work, we assume that the X-ray images are calibrated for their intrinsic parameters and that the X-ray images are corrected for distortion. If multiple $\mathrm{X}$-ray images are used, they are all registered to a common reference frame. Therefore, the goal of a $3 \mathrm{D}-2 \mathrm{D}$ registration is to compute the rigid transformation $T$ that relates the coordinate frame of the CT volume with the reference coordinate frame of the X-ray images. In the following, we focus on the derivation based on the $q$ th X-ray image (where $q=1, \ldots, Q$ ) and its associated DRR.

Let us denote the values of the X-ray image $(V)$ as $v(x)$ and the corresponding values of the $\operatorname{DRR}(U)$ created from the $\mathrm{CT}$ volume given the current transformation estimation as $u(x ; T)$. In this work, we regard the image values $v(x)$ and $u(x ; T)$ as random variables with associated probability density functions $p(v(x))$ and $p(u(x ; T))$, respectively. The joint probability density function of these two random variables is $p(v(x), u(x ; T))$. The conditional probability density function of $v(x)$ given the values of $u(x ; T)$ is expressed as $p(v(x) \mid u(x ; T))$.

The mutual information of two random variables is derived from the entropy values of the variables:

$$
S_{M I}^{q}(V, U, T)=H(V)+H(U)-H(V, U)=H(V)-H(V \mid U)
$$

After some replacement, we can write Eq. 1 as:

$$
S_{M I}^{q}(V, U, T)=\iint p(v(x), u(x ; T)) \log (p(v(x) \mid u(x ; T))) d v d u+H(V)
$$

The optimal estimation of the rigid transformation can then be obtained by:

$$
\hat{T}=\underset{T}{\arg \max } \sum_{q=1}^{Q} S_{M I}^{q}(V, U, T)
$$

Eq. 3 is the standard registration framework using maximization of mutual information. Histogram-based method [2] as well as Parzen window based method 
[1] have been proposed to compute the mutual information. It is known that the standard mutual information measure only takes intensity values into account without considering spatial information and its robustness are questionable. It can be shown using Kullback-Liebler bound [9] that:

$$
S_{M I}^{q}(V, U, T) \geqslant \iint p(v(x), u(x ; T)) \log (q(v(x) \mid u(x ; T)) d v d u+H(V)
$$

where $q(v(x) \mid u(x ; T))$ is an arbitrary variational distribution. We call the right side of Eq. 4 the variational approximation to mutual information (VA-MI) and denote it as $S_{V A-M I}^{q}(V, U, T)$. The approximation is exact if $q(v(x) \mid u(x ; T)) \equiv$ $p(v(x) \mid u(x ; T))$.

As we are dealing with discrete images, the values $v(x=(i, j))$ and $u((x=$ $(i, j)) ; T)$ that we observe from the images can be regarded as random samples from $p(v(x), u(x ; T))$. Note that $H(V)$ in Eq. 4 does not depend on $T$. Ignoring this constant term, we can further approximate $S_{V A-M I}^{q}(v(x), u(x ; T))$ by its sample estimate:

$$
S_{V A-M I}^{q}(V, U, T) \approx \frac{1}{I \times J} \sum_{i=1}^{I} \sum_{j=1}^{J} \log (q(v(i, j) \mid u((i, j) ; T)))
$$

where $I \times J$ is the pixel size of the X-ray image.

Using Eq. 5, we actually convert the maximization of mutual information to an optimal labeling problem in which the labels are the conditional intensity values $(v(i, j) \mid u((i, j) ; T))$. Such a problem can be effectively solved using a Markov random field model. Interestingly, we found that a similar form as expressed by Eq. 5 could also be derived using a maximum likelihood approach as done by Roche et al. 11. However, compared to their derivation, our derivation is much simpler and more intuitive.

\subsection{Realization of the Variational Approximation}

Markov Random Field Theory. Markov random field theory has been used extensively in image restoration, segmentation, object recognition and matching [10. In what follows, the basic concepts of the MRF are reviewed for completeness. For rigorous expositions, one may refer to [10].

Let $L=\{(i, j): 1 \leqslant i \leqslant I, 1 \leqslant j \leqslant J\}$ be an $I \times J$ integer lattice; then $D=\left\{D_{i, j} ;(i, j) \in L\right\}$ denotes a family of random variables, i.e., a random field, defined on $L$. A system $d$ can be viewed as a discrete sample realization of $\mathrm{D}$ assuming certain state on each pixel site. $\mathbf{d}=\left\{d_{1,1}, d_{1,2}, \cdots, d_{I, J}\right\}$ is referred as a configuration of $D$. The complete set of all the configuration is denoted as $\mathbf{D}$.

Definition 1: A neighborhood system for $L$ is defined as $\mathbf{N}=\left\{N_{i, j}^{r} ;(i, j) \in L\right\}$, where $N_{i, j}^{r}$ is the set of sites around $(i, j)$ and is defined as follows:

$$
N_{i, j}^{r}=\left\{\left(i^{\prime}, j^{\prime}\right)\left|\left(i^{\prime}, j^{\prime}\right) \in L,\left(i^{\prime}, j^{\prime}\right) \neq(i, j),\right|\left(i^{\prime}, j^{\prime}\right)-(i, j) \mid \leqslant r\right\}
$$

where $r$ is a positive integer that determines the size of the neighborhood system. 
Definition 2: A clique $c$ is a subset of $L$, for which every pair of sites is a neighbor. Single pixels are also considered cliques. The set of all cliques related with the pixel site $(i, j)$ is denoted by $C_{i, j}$.

Definition 3: $D$ is a Markov random field with respect to (w.r.t.) the neighborhood system $\mathbf{N}$ if and only if:

$$
p(\mathbf{d})=\frac{1}{z} e^{-E(\mathbf{d})}
$$

where $p(\mathbf{d})$ is called a Gibbs measure of $\mathbf{d} ; z$ is a normalization constant called the partition function and $E(\mathbf{d})$ is the energy function of the form:

$$
E(\mathbf{d})=-\sum_{i, j}^{I, J} \sum_{c \in C_{i, j}} W_{c}\left(d_{i, j}\right)
$$

where $W_{c}$ is called the clique potential. Generally, $W_{c}$ is a function of the cliques around the site under consideration.

Realization of the Variational Approximation Using a MRF. To estimate the conditional distribution $q(v(x) \mid u(x ; T)$ ), knowing the exact values of $v(i, j)$ and $u((i, j) ; T)$ are not important. We are more interested in knowing the conditional difference between $v(i, j)$ and $u((i, j) ; T)$. Having this knowledge and using the concept of Gibbs measure, we can approximate the conditional distribution $q(v \mid u)$ using a MRF w.r.t. the neighborhood system $\mathbf{N}$ defined on the lattice $L=\{(i, j): 1 \leqslant i \leqslant I, 1 \leqslant j \leqslant J\}$ of the X-ray image.

However, until now we still can not directly compare $v(i, j)$ to $u((i, j) ; T)$ at each pixel site because there are inherent differences between the X-ray image and the associated DRR. In this paper, we propose to use a local normalization to circumvent this problem. The rationale behind it is that in a local region the intensity differences between different sites are mainly caused by the imaged object, if no external object presents in the field-of-view.

Definition 4: A local region of size $r$ for the pixel site $(i, j) \in L$ is the set of sites defined by:

$$
R_{i, j}^{r}=\left\{\left(i^{\prime}, j^{\prime}\right)\left|\left(i^{\prime}, j^{\prime}\right) \in L,\right|\left(i^{\prime}, j^{\prime}\right)-(i, j) \mid \leqslant r\right\}
$$

The local normalization of both the X-ray image and the associated DRR is then performed as follows:

$$
\bar{v}(i, j)=\frac{v(i, j)-m_{v}\left(R_{i, j}^{r}\right)}{\sigma_{v}\left(R_{i, j}^{r}\right)} ; \text { and } \bar{u}((i, j) ; T)=\frac{u((i, j) ; T)-m_{u}\left(R_{i, j}^{r}\right)}{\sigma_{u}\left(R_{i, j}^{r}\right)}
$$

where $m_{v}\left(R_{i, j}^{r}\right), \sigma_{v}\left(R_{i, j}^{r}\right)$ and $m_{u}\left(R_{i, j}^{r}\right), \sigma_{u}\left(R_{i, j}^{r}\right)$ are the mean value and the standard deviation calculated from the intensity values of all sites in the local region $R_{i, j}^{r}$ of the X-ray image and of the associated DRR, respectively.

We can now model the difference image

$$
s((i, j) ; T)=\bar{v}(i, j)-\bar{u}((i, j) ; T)
$$


as a MRF w.r.t. the neighborhood system $\mathbf{N}$ defined on the lattice $L=\{(i, j)$ : $1 \leqslant i \leqslant I, 1 \leqslant j \leqslant J\}$. According to the relationship between the probability measure and the energy function of a MRF at a single site, we have:

$$
\begin{aligned}
& \log q(v(i, j) \mid u(i, j) ; T) \approx \log p(s((i, j) ; T) ; \text { and } \\
& \log p\left(s((i, j) ; T)=-E\left(s((i, j) ; T)=-\sum_{c \in C_{i, j}} W_{c}(s(i, j) ; T)\right.\right.
\end{aligned}
$$

We can further expand the clique potentials in Eq. 12 according to the clique size. In this work, we only consider the cliques of size up to two. Using such an approximation, we derive a new similarity measure. We call it the MRF model based variational approximation to mutual information (MRF-VA-MI) and denote it as $S_{M R F-V A-M I}^{q}(V, U, T)$. It has the form:

$$
\begin{aligned}
& -S_{V A-M I}^{q}(V, U, T) \approx S_{M R F-V A-M I}^{q}(V, U, T)=\sum_{i, j}^{I, J} W_{c}(s((i, j) ; T)) \\
& +\sum_{i, j}^{I, J} \frac{1}{\operatorname{card}\left(N_{i, j}^{r}\right)} \cdot \sum_{\left(i^{\prime}, j^{\prime}\right) \in N_{i, j}^{r}} W_{c}\left(s((i, j) ; T), s\left(\left(i^{\prime}, j^{\prime}\right) ; T\right)\right)
\end{aligned}
$$

where $S_{V A-M I}^{q}(V, U, T)$ is negative because mutual information is maximized, whereas energy must be minimized. The first term of the rightist side is the potential function for single-pixel cliques and the second term is the potential function for all other pairwised cliques. $\operatorname{card}\left(N_{i, j}^{r}\right)$ means to compute the number of pixels in neighborhood $N_{i, j}^{r}$.

The selection of the potential function in Eq. 13 is a critical issue in MRF modeling [10]. By choosing different potential functions, we can derive different similarity measures. Here we give two examples of deriving previously published well-known similarity measures based on the present framework.

1. Sum-of-Squared-Difference (SSD): SSD can be derived from Eq.14 by setting:

$$
\begin{aligned}
& W_{c}(s((i, j) ; T))=[s((i, j) ; T)]^{2} \\
& W_{c}\left(s((i, j) ; T), s\left(\left(i^{\prime}, j^{\prime}\right) ; T\right)\right)=0
\end{aligned}
$$

2. Pattern Intensity: the pattern intensity proposed in [12] has the following form:

$$
P_{r, \sigma}=\sum_{i, j} \frac{1}{\operatorname{card}\left(N_{i, j}^{r}\right)} \sum_{\left(i^{\prime}, j^{\prime}\right) \in N_{i, j}^{r}} \frac{\sigma^{2}}{\sigma^{2}+\left[s\left(\left(i^{\prime}, j^{\prime}\right) ; T\right)-s((i, j) ; T)\right]^{2}}
$$

where $r$ and $\sigma$ are two parameters to be experimentally determined. It can be derived from the present framework by using following pairwised clique potential function:

$$
\begin{aligned}
& W_{c}(s((i, j) ; T))=0 \\
& W_{c}\left(s((i, j) ; T), s\left(\left(i^{\prime}, j^{\prime}\right) ; T\right)\right)=-\frac{1}{1+\frac{\left[s\left(\left(i^{\prime}, j^{\prime}\right) ; T\right)-s((i, j) ; T)\right]^{2}}{\sigma^{2}}}
\end{aligned}
$$

In this work, we simply use following potential functions to derive a new similarity measure. We name the newly derived similarity measure as the pairwised 
MRF model based variational approximation to mutual information (PW-MRFVA-MI) and denote it as $S_{P W-M R F-V A-M I}^{q}(V, U, T)$ :

$$
\begin{aligned}
& W_{c}(s((i, j) ; T))=[s((i, j) ; T)]^{2} \\
& W_{c}\left(s((i, j) ; T), s\left(\left(i^{\prime}, j^{\prime}\right) ; T\right)\right)=\left[s((i, j) ; T)-s\left(\left(i^{\prime}, j^{\prime}\right) ; T\right)\right]^{2}
\end{aligned}
$$

\subsection{Implementation Details}

To accelerate the registration process, we exploit a spline-based multi-resolution $3 \mathrm{D}-2 \mathrm{D}$ registration scheme 13 . A cubic-splines data model is used to compute the multi-resolution data pyramids for the CT volume, the X-ray images, the DRRs, as well as for the gradient and the Hessian of the PW-MRF-VA-MI. The registration is then performed from the coarsest resolution until the finest one. At each resolution level, the size of the local region for the normalization is always equal to that of the neighborhood system used in Eq. 13. And to improve the capture range, we use two different sizes of neighborhood systems: $r=15$ and $r=3$. The PW-MRF-VA-MI with the bigger neighborhood system is first minimized via a Levenberg-Marquardt non-linear least-squares optimizer. The estimated $\hat{T}$ is then treated as the starting value for optimizing the PW-MRF-VA-MI with the smaller neighborhood system.

\section{Experimental Results}

We conducted two studies on X-ray and CT datasets of a plastic phantom and a cadaveric spine segment. The data sizes, the original data resolution, the start and the end resolutions of the X-ray and the CT datasets are summarized in Table 1. The ground truth transformations of both datasets were obtained by performing paired-point matchings on implanted fiduaial markers. The phantom was custom-made to simulate a good condition. In contrast, the quality of the X-ray images for the cadaveric spine was poor and there were projections of interventional instruments presented.

Using the datasets of both objects downsampled until the start resolution, we first compared the behavior of the PW-MRF-VA-MI to those of a MI-based measure using a histogram-based implementation [2] and of a similarity measure introduced in [13], which is a global normalization based SSD. The results are presented in Fig. 1. It was found that all similarity measures had similar behavior when tested on the phantom dataset but different behavior when tested on the spine segment dataset. The PW-MRF-VA-MI shows a superior behavior compared to others. More specifically, all curves of the PW-MRF-VA-MI have clear minima and are smoother than those of others. It also shows that using bigger neighborhood system, which is equivalent to incorporate wider range of spatial information, leads to smoother energy function whereas using smaller neighborhood system results in higher accuracy.

The second study was performed only on spine segment dataset to evaluate the performance of the registration scheme using the PW-MRF-VA-MI. In this 

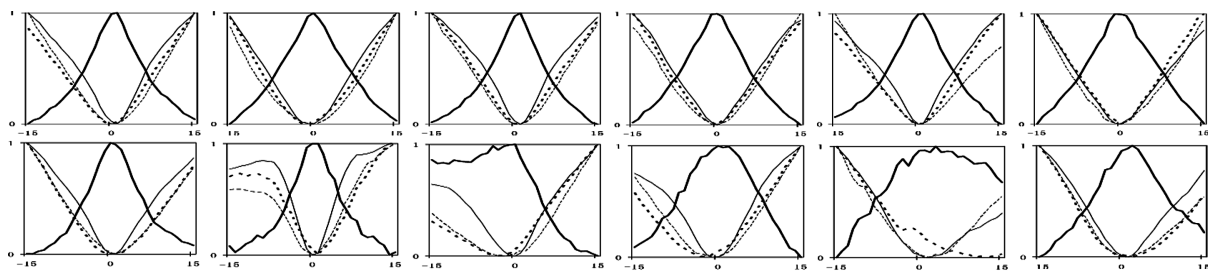

$-\cdots \cdot-\cdot S S D$

Fig. 1. Probe through the minimum of similarity measures on the phantom data (the first row) and on the spine data (the second row). The ordinate shows the value of similarity measures normalized to the range $[0.0,1.0]$, which are given as functions of each parameter in the range of $\left[-15^{\circ}, 15^{\circ}\right]$ or $[-15 \mathrm{~mm}, 15 \mathrm{~mm}]$ away from its ground truth (the first three columns represent the translational probe along $\mathrm{X}, \mathrm{Y}$ and $\mathrm{Z}$ axis, respectively; the last three columns represents the rotational probe along each axis).

Table 1. Data specifications

\begin{tabular}{l|c|c|c|c}
\hline \multicolumn{5}{c}{ CT Data Specification } \\
\hline test object & rows $\times$ columns $\times$ slices & data res. $\left(\mathrm{mm}^{3}\right)$ & start res. $\left(\mathrm{mm}^{3}\right)$ & end res. $\left(\mathrm{mm}^{3}\right)$ \\
\hline phantom & $512 \times 512 \times 93$ & $0.36 \times 0.36 \times 2.5$ & $2.88 \times 2.88 \times 2.5$ & $2.88 \times 2.88 \times 2.5$ \\
\hline cadaver & $512 \times 512 \times 72$ & $0.36 \times 0.36 \times 1.25$ & $2.88 \times 2.88 \times 1.25$ & $2.88 \times 2.88 \times 1.25$ \\
\hline \multicolumn{5}{c}{ X-ray Data Specification } \\
\hline test object & width $\times$ height $\times$ images & data res. $\left(\mathrm{mm}^{2}\right)$ & start res. $\left(\mathrm{mm}^{2}\right)$ & end res. $\left(\mathrm{mm}^{2}\right)$ \\
\hline phantom & $768 \times 576 \times 2$ & $0.39 \times 0.39$ & $3.12 \times 3.12$ & $3.12 \times 3.12$ \\
\hline cadaver & $768 \times 576 \times 2$ & $0.39 \times 0.39$ & $3.12 \times 3.12$ & $3.12 \times 3.12$ \\
\hline
\end{tabular}

Table 2. Study results using the datasets of cadaveric spine segment

\begin{tabular}{l|c|c|c|c|c|c}
\hline absolute parameter range $\left({ }^{\circ}, \mathrm{mm}\right)$ & $(2,2)$ & $(4,4)$ & $(6,6)$ & $(8,8)$ & $(10,10)$ & $(12,12)$ \\
\hline average of the initial mTRE $(\mathrm{mm})$ & 2.3 & 4.6 & 6.9 & 9.2 & 11.5 & 13.5 \\
\hline success percentage & 100 & 100 & 100 & 99 & 95 & 85 \\
\hline average of the final mTRE $(\mathrm{mm})$ & 0.8 & 0.8 & 0.8 & 0.8 & 0.8 & 0.8 \\
\hline
\end{tabular}

study, we perturbed the ground truth by randomly varying each parameter in the range of $\left[-2^{\circ}, 2^{\circ}\right]$ or $[-2 \mathrm{~mm}, 2 \mathrm{~mm}]$ to get 200 positions, and then another 200 positions in the rage of $\left[-4^{\circ}, 4^{\circ}\right]$ or $[-4 \mathrm{~mm}, 4 \mathrm{~mm}]$, and so on until the range of $\left[-12^{\circ}, 12^{\circ}\right]$ or $[-12 \mathrm{~mm}, 12 \mathrm{~mm}]$. We then performed the registration starting from these perturbed positions and counted the success rate. Using a method similar to that reported in [14, we regarded a registration as successful if the mean target registration errors (mTRE) evaluated on the fiducial markers was smaller than $1.5 \mathrm{~mm}$. The capture range was defined as the average of the initial mTRE when a $95 \%$ success rate is achieved. The study results are presented in Table 2. When the absolute parameter range is $\left(12^{\circ}, 12 \mathrm{~mm}\right)$, the average CPU time tested on a $3.0 \mathrm{GHz}$ Pentium machine was 26.7 seconds. It was found that the capture range of the PW-MRF-VA-MI was much larger than those reported in [7] and in [14, although the attained accuracy was lower than that reported 
in [14. This might be explained by the large inter-slice distance $(2.5 \mathrm{~mm}$ in this work vs. $0.31 \mathrm{~mm}$ in [14]) and the region outliers in the X-ray images.

\section{Conclusions}

In this paper, we derived a novel information and Markov random field theory based similarity measure, the MRF model based variational approximation to mutual information, based on the Kullback-Leibler bound. The newly derived similarity measure enabled us to effectively incorporate spatial information into a 3D-2D registration. Results from the experiments performed on the datasets of a plastic phantom and of a cadaveric spine segment show that the newly derived similarity measure has a larger capture range than those have been previously reported and attains satisfactory accuracy.

\section{References}

1. Wells, W., Viola, P., et al.: Multi-model volume registration by maximization of mutual information. MedIA 1, 35-51 (1996)

2. Maes, F., Collignon, A., et al.: Multimodality image registration by maximization of mutual information. IEEE Trans. Med. Imaging 16, 187-1998 (1997)

3. Pluim, J.P., et al.: Mutual information based registration of medical images: a survery. IEEE Trans. Med. Imaging 22, 986-1004 (2003)

4. Pluim, J., et al.: Image registration by maximization of combined mutual information and gradient information. IEEE Trans. Med. Imaging 19, 809-814 (2000)

5. Rueckert, D., Clarkson, M.J., et al.: Non-rigid registration using higher-order mutual information. SPIE Medical Imaging: image processing 3979, 438-447 (2000)

6. Sabuncu, M.R., Ramadge, P.J.: Spatial information in entropy-based image registration. In: Gee, J.C., Maintz, J.B.A., Vannier, M.W. (eds.) WBIR 2003. LNCS, vol. 2717, pp. 132-141. Springer, Heidelberg (2003)

7. Russakoff, D.B., Tomasi, C., et al.: Image similarity using mutual information of regions. In: Pajdla, T., Matas, J(G.) (eds.) ECCV 2004. LNCS, vol. 3023, pp. 596-607. Springer, Heidelberg (2004)

8. Gan, R., Chung, A.C.S.: Multi-dimensional mutual information based robust image registration using maximum distance-gradient-magnitude. In: Christensen, G.E., Sonka, M. (eds.) IPMI 2005. LNCS, vol. 3565, pp. 210-221. Springer, Heidelberg (2005)

9. Barber, D., Agakov, F.V.: The IM algorithm: a variational approach to information maximization. In: NIPS 2003, vol. 16. MIT Press, Cambridge (2004)

10. Li, S.Z.: Markov random field modeling in computer vision. Springer, Heidelberg (1995)

11. Roche, A., Malandain, G., Ayache, N.: Unifying maximum likelihood approaches in medical image. Int. J. Image Systems and Technology 11, 71-80 (2000)

12. Weese, J., et al.: An approach to $2 \mathrm{D} / 3 \mathrm{D}$ registration of a vertebra in $2 \mathrm{D}$ x-ray fluoroscopies with 3D CT images. In: Troccaz, J., Mösges, R., Grimson, W.E.L. (eds.) CVRMed-MRCAS 1997, CVRMed 1997, and MRCAS 1997. LNCS, vol. 1205, pp. 119-128. Springer, Heidelberg (1997)

13. Jonić, S., Thévenaz, P., et al.: An optimized spline-based registation of a 3D CT to a set of C-arm images. nt. J. Biomed. Imaging Article ID 47197, 1-12 (2006)

14. von de Kraats, E.B., Penney, G.P., et al.: Standardized evaluation methodology for 2-D-3-D registration. IEEE Trans. Med. Imaging 24, 1177-1189 (2005) 